\title{
A Digital Micro-Game Approach to Improve the Learning of Hand-Weaving Art and History
}

\author{
https://doi.org/10.3991/ijet.v16i08.19795 \\ Supaporn Chai-Arayalert $\left.{ }^{(}\right)$, Supattra Puttinaovarat \\ Prince of Songkla University, Surat Thani, Thailand \\ supaporn.chaiepsu.ac.th
}

\begin{abstract}
This research focuses on the young people and an issue of national handicraft preservation in the form of southern style hand weaving. A game is used in the study on arts and cultures as a learning medium optimized for the young people who play an important role in preserving the traditional handicraft being at the verge of extinction. The "Exploring Na Muen Sri" was developed based on digital micro-game and Person-Artefact-Task model as well as the game production with the purpose of creating the learning media and a simulation containing new knowledge about hand-weaving art and history that is suitable for the young people. The case study was on the weaving history of Na Muen Sri Community located in the southern Thailand. An experiment was conducted with undergraduate students to explore the effectiveness of the proposed approach in the field of cultural study. The results show that the game can effectively enhance players' cognitive growth along with cultural awareness. It can be concluded that the simulated learning environment created in the digital game enables to bring about comprehension supporting the lifelong learning of hand weaving art and history while simultaneously preserving the local wisdom of hand weaving of fabrics.
\end{abstract}

Keywords - Digital micro-game; lifelong learning; Hand-weaving; Person-Artefact-Task model

\section{Introduction}

The advancement of lifelong learning is consistent with the rapid growth of digital technological development [1], [2]. A Digital Game (DG), one of the learning media technologies, has been very popular due to its significant feature of imaginative creation, challenge and competition that not only attract players but also motivate them. Apart from being applicable for learning as well as learning enhancement and distinctions among players, the DG is a development tool that transforms learners' behaviors, and heightens lifelong learning and self-directed learning. The report of [3] on the statistic of using the internet in Thailand reveals that Thai people of ages 16-64 use the internet 9 hours/day, in which almost 2 hours are spent on games: the use of game online application has increased by $67 \%$. That is, the amount of DG players among Thais continuously increases, mostly on mobile phones ( $88 \%$ ) followed by computers (46\%). The survey conducted on the situation of playing online games among Thai youth in 2019 
as reported by [4] shows that $88.33 \%$ of the sampled group of 3,000 high school students/ equivalent group used to play online games, with $65 \%$ used to playing every day, on average 3-5 hours/day.

Thailand arts and cultures are the country's identity exposing its background, especially the local weaving of fabrics that represents the local wisdom derived from regional accumulated learning and transmitting it from generation to the next [5]. That is, the local weaving of fabrics symbolizes the local wisdom as ongoing integrated knowledge, as the fabric clearly shows the uniqueness of cultural mixture while being a medium in telling stories, beliefs, lifestyle as well as the existence of each era [5]. In addition, the weaving of fabrics is also a combination of various sciences and arts that are worth preservation. The illustration of hand weaving of fabric can be in a form of temporary exhibitions or traditional museums or online information presentations [5]. However, the study of transmission, continuation, and preservation of the hand weaving art that can lead to sustainability is considered a challenge, especially when being conducted among the young people who are the main drive for such preservation. For instance, if the applied technology and methods used for learning media are unattractive or non-innovative and lack technology for learning and supporting the preservation and sustainability, it can lead to difficulties in the dissemination and access to the media. Such hindrances affect the media users, particularly the young people who have to put more effort in the learning [5], [6]. On the other hand, the simulation in digital games is able to transform art and cultural contexts into real experiences, while opening doors to users to the cultural value of local wisdoms. Viewers can access the heritage or art and cultural information with lesser risk of damage when compared to the real approach. Moreover, knowledge transmission of a traditional form might cause the lack of real experience, the one that can create the learning motivation.

DGs as media have been used in various aspects, such as education, training, medication, and tourism. Nevertheless, studies employing DG in the field of intangible culture perspective for art and cultural learning, for example, oral traditions, performing arts, local knowledge, and traditional skills, are still very rare (e.g. [7], [8]). Furthermore, DG development consumes large resources and time while players have to possess a high efficiency device along with internet access in order to be able to smoothly play the games. With such limitations, a Digital Micro-Game (Digital MG) approach is preferred for the development in this current research framework.

The targeted group for this research was the young $19-22$ years old people, who are considered a significant force in art and cultural preservation, and they were influenced by technology that affects their learning transformation [9], [10]. Apart from the learning gained, these young people also needed to enjoy the process [11], [12]. In addition, they were found to have behaviours in line with the expression "The internet in its pocket generation" [13], or in other words, they possessed the device that enabled them to always connect with the internet. They could learn, work and communicate with others at all times and it was more interesting to them if they were able to combine the technology with their daily activities. It has been quite difficult to use the traditional learning media nowadays to create satisfying learning experiences that can respond to the young people's behaviors and interests [14]. Thus, simulation of environmental development to upgrade the weaving fabric art and culture becomes a motive to create 
interest among the youth so that they can gain knowledge through practical and actionbased learning experiences.

The objective of this paper was to design and develop a Digital MG as a practical learning medium for youth so that they could have new learning experience on the handweaving art and history. The target was to create and disseminate knowledge and understanding on southern weaving art and culture, which is a cultural heritage worth preservation; and, simultaneously, to enable the young people to know how to preserve local wisdom of hand-weaving fabrics as well as to support life-long learning about hand-weaving art and history.

\section{Related Work}

Digital games are about using multimedia technology to function under the practical environment where players enjoy different tasks and challenges to complete the mission. The games are able to motivate or stimulate players to pay more attention to the stories. Playing the game requires a device with which the players act according to the game rules and goals [15]-[17]. There are important components to be considered in designing a DG, which include goals, rules, competitions, challenges, imagination, and enjoyment [4], [16], [17]. The DG must be able to stimulate players to utilize their abilities, skills, and techniques and that they are able to conduct self-problem solving while playing the game, whereas the learning outcome instantly reflexes [4], [17]. The utilization of DG as a learning medium is to interpolate the context needed to be transmitted in a form of game and, sometimes, it allows players to participate in the practice while maintaining the practical environment which helps learners enjoy, feel the challenge and possess more drive to learn [11], [12].

It has become more complex to conduct DG development. The complexity includes playing modes, rules, requirement to get more players to participate as solo becomes less fun, etc. The focus seems to be on entertainment instead of learning. In order to succeed and reach the goal, players might need to use more skills and spend a long time in playing the game, which means they have to continuously play, not to mention that the playing needs a special device. Subsequently, the DG development takes a long time and uses resources with a high cost along with a lot of teamwork. Thus, digital micro-game (DMG) approach seems to be a better choice for learning media development. The micro-game is a small game often within another game, and sometimes in application software or on a display of some form of hardware. A micro-game is composed of more game play elements than the main game, may be optional, and is often smaller or more simplistic than the game in which it is contained [6], [19]. As for this research, the micro-game is a digital small game, and within it, also contains small activities making it easy for players to achieve some aspects of learning objectives. The players can spend only a short time in the game and it is unnecessary that they possess a specific skill to play such game, whereas the game can be applied with their work or daily life [19]-[21]

DMG approach helps ease off the complex context by being in an easy-to-learn format [22], [23]. Applying the DMG approach in this research is aimed to (1) develop a 
game with lesser utilization of resources and at a lower cost than other DGs; (2) reduce the development steps; and, (3) be able to implement quickly. The result of the aforementioned qualifications makes the game development consistent with young people's behaviours of paying attention to things only for a short time, because of the digital technological advancement along with the quick access, only at a fingertip, to information [6], [19]. Moreover, the DMG can transform the uninteresting cultural context into an interesting one as the cultural information can be released in minimal snippets to gain the appropriate level of understanding at each point during a game narratives and reducing the youth learning limitations due to its mechanism. DMGs can unveil information about hand-weaving art and history of the smallest possible form to ensure that the users focus exactly on the desired objective. Thereby, the players' confusion and misdirection are prevented by providing excess information; meanwhile, an iteration of new data can be applied directly. Therefore, in this paper, a DMG approach for development of art and cultural learning as well as of history of southern hand weaving is formed by combining virtual and reality in historical time.

\section{Case Study of Na Muen Sri Community Enterprise}

Na Muen Sri Community Enterprise, the research case study, is in Trang province, in southern peninsular Thailand, where weaving wisdom and art are preserved through an exhibition in the traditional museum at Na Muen Sri Learning Center Building, aiming to disseminate the textile historical background, uniqueness, and elegance. Nevertheless, learning barriers exist, including (1) time consumed by individuals in travelling to the center; (2) the presented information being difficult to comprehend; (3) non-stimulating activities to attract learners; etc. In addition, previous studies disclose various problems with students/learners [24], [25], for instance, boring teaching and learning media, teachers' uninteresting methods or lack of teaching documents, or non-historical references or non-explanations of historical events, as well as difficulties in memorizing historical facts and understanding the historical environment [24], [25]. This is consistent with [25], who find a lack of methods or approaches in knowledge presentation through digital along with the non-existence of DMG design and development for youth in learning as well as non-awareness creation to preserve arts and cultures. Youth have an insufficient opportunity to access knowledge, understanding and attention regarding local history of traditional hand-weaving art. This study is a continuation of the abovementioned paper with design and development of digital micro-game based learning approach to learn the southern hand-weaving art and culture by informal as well as selfdirected learning. Multimedia and interactive technology can efficiently enhance the learning among youth while creating the learning drive for history, arts and culture through interesting teaching media.

\section{$4 \quad$ Research Framework}

The objective of this research framework was to create understanding, design and develop a DMG that enables to present, transmit and disseminate the knowledge to 
young players. This paper employed Person-Artefact-Task (PAT) Model for DMG development through the Flow Theory [26], the study of players' behaviours upon doing activities, along with their willingness and participation. If players clearly comprehended the activity objectives, they would be able to promptly solve problems. The ability in problem solving while conducting the activity/event could make players satisfied, which further led to action and awareness, concentration, sense of control over an activity, a loss of reflection, self-consciousness and experience on the activity .PAT model is emphasized prior to gaining knowledge and experience of a person performing the task with the artefact within computer-mediated environments [26]. In designing DMG, three components (Person-Artefact-Task) should be considered and captured in how players deal with challenges in the game [27]. It is critical that a game system offers players/learners such challenges that are compatible with their skill levels, so the possibility of experiencing flow is high [27]. This will lead the learners to increase their level of learning, motivate their exploration behaviours and also raise cultural awareness [26], [28], [29].

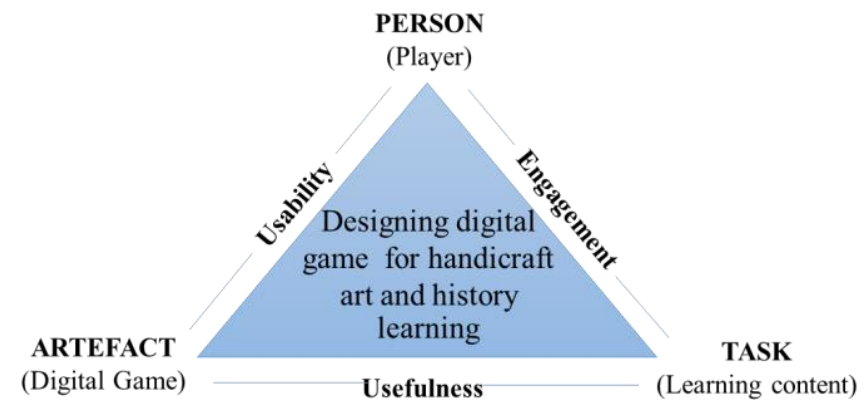

Fig. 1. Research framework (adapted from [26], [28])

Figure 1 describes (1) a person, which means a player or learner for whom, in DMG design, the player's skill must be considered so that the playing can be stress free since difficult games might lead to stressfulness; in contrast, too-easy games can lead to boredom, bare in mind that the game's main objective is the players' learning to reach the targeted level and game objective; (2) artefact, which is a game design of informal learning media being optimized with the targeted group and the set learning content, that is, the game must provide both knowledge and fun; and, (3) tasks, which are the learning contents and the important parts in game development in which the design must have a clear content while being suitable with the game patterns and players, and simultaneously be consistent with the set learning objectives.

\section{Designing Digital Micro-Game for "Exploring Na Muen Sri"}

The research framework in Figure 1 was applied to design and develop "Exploring $\mathrm{Na}$ Muen Sri" game by considering the following aspects: 
Designing and developing the game must be focused on optimal usability to the players or the interactions between players and the digital micro-game. Also, the focus must be on game genre, mechanism, aesthetics, and technology [18], [26], [30] whereas the game development was composed of its genre on the historical content and adventurous type. Moreover, the game must be played continuously according to its rules. Similarly, the game mechanisms were its procedures and rules in which the mechanics described the goal, "how players can and cannot try to achieve, and what happens when they succeed". As the game was in a single-player mode, the player must serve as an actor who travelled, explored and worked on a set task within the assigned time. The player was arranged to be in an assumed world and to play the role as set in the content. Moreover, he/she could make a decision and freely play the game. The mechanics design helped players learn a story through the game as the information was released in the smallest possible pieces to convey the appropriate level of understanding at each point in a game's narrative. The game would have gradually increased level of difficulty based on the duration of game-playing. As for game aesthetics, it was a design on an artistic movement in regards to visual, audio, and atmosphere that was user friendly and consistent. Eventually, the technology for 3D game development was in the Window OS platform.

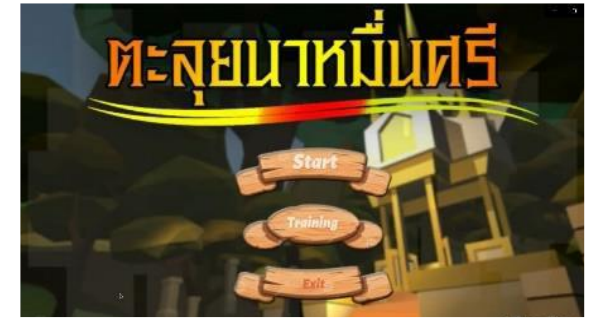

a) Playing section

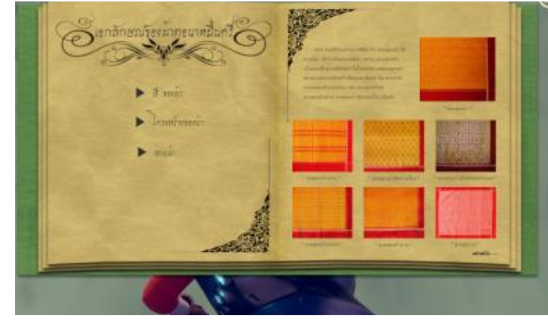

b) Learning section

Fig. 2. Example of "Exploring Na Muen Sri” game

The developed game designates a player to have a role of "Thong" who wonders and wants to know about Na Muen Sri weaving fabric. The player/Thong conducts an exploration on the source of weaving area in a form of visual environment by talking to the locals who give him/her information on each aspect. The player has self-control of the game and goes through each challenge until the final point before he/she can answer questions regarding the Na Muen Sri weaving fabric. The game is divided into (1) introduction; (2) game playing; and (3) game ending, and the player must go through all three challenges in order to collect the entire information of the fabric prior to entering into a question-answer mode and completing the game. Again, the game objective is to enhance learning and heighten motivation while becoming a transmittal tool on knowledge and entertainment that further stimulates an individual's interest in handweaving art and history. 
Figure 2(a) demonstrates a playing section in a form of adventure, yet with knowledge accumulation as a player must go through each challenge of Na Muen Sri Weaving Learning Centre. Here, the player must complete the designated task and answer all questions correctly to collect his/her scores. One question/answer is one mark and the answering must be ongoing until the 10 questions are completed, which means that the game is over.

In designing and developing the game, the focus is on learning content and its usefulness or "the interaction between digital micro-game and learning content" in order to ensure the declaration knowledge of hand-weaving history and art is transformed into DMG, the learning tool/medium under entertaining environment [18], [26], [30] . Transforming from the traditional learning content to $3 \mathrm{D}$ virtual environment is comprised of hand weaving background, producing source, knowledge on weaving art, unique design, raw material and weaving tool, as well as a producing process. The game design is a simulation of 3D Art and Cultural Exhibition Centre with "Thong" as a host of the game through communication and interaction with other characters. The game features can be summarized as: the virtual exhibition of the hand-weaving art and history of "Na Muen Sri", a sort of storytelling through the main actors being set as "the first person game" where players are able to have real experience by playing the roles as assumed. The pattern is a treasure hunting game about handicraft art and history that can be played in the Windows platform where players are obliged to go through each set challenge. The learning target is the transmission of local cultures presented in a digital format to enhance learning and driving that, simultaneously, becoming a tool for knowledge transmission, entertainment as well as stimulating player's interest in local art, history and preservation. Thus, the game becomes a combination between the virtual exhibition of Na Muen Sri and motivation creation in missions through the development of a meaningful learning environment for cultural hand-weaving art.

The game storytelling starts by using a content of the Na Muen Sri weaving group community with a player taking a role of a character in the game. The player plays a role as set and decides whether to complete or cancel the designated activity. Prior to playing, the player must enter a mode of "how to play" to understand the target (the exploration and search for information on hand weaving of fabrics and its related knowledge) in which the player plays a role of a boy residing and growing up in $\mathrm{Na}$ Muen Sri Sub-district, Trang province, one of the famous fabric sources in Thailand, the sub-district being used as the location of the game. Each scene is designated for player to work on the search mission "the puzzles of local hand-weaving art and history" (the feature of hand-weaving art, history and its genres for a beginner' friendly way). In each challenge, the player must collect knowledge from other actors so that, at the end of all set challenges, such player is able to learn the history, uniqueness, materials and tools as well as knowledge on Na Muen Sri weaving of fabrics.

In designing a game challenge to be suitable for players and assuring that the players would not find such challenge too easy or too difficult and stop halfway, rewards are arranged once the players properly succeed to overcome the challenge. The examples of the challenge include exploring the virtual exhibition by means of looking around, investigating certain tasks and the puzzles of cultural knowledge of hand-weaving 
textiles, and remembering knowledge from previous scene to fulfil the tasks. Figure 2 (b) is a learning section from Na Muen Sri weaving simulation learning centre.

In order to conduct self-knowledge, the design and development are considered from the engagement or "the interaction between a player and a learning content" by using the game to drive players so that they participate in the playing while ascending a positive feeling toward the game learning [15], [18], [26], [30]. An interesting game can always have an effect on a player to pay attention and participate in activities to reach the final point so that, subsequently, both direct and indirect learning occur. As for this research game, there is featuring and storytelling that focus players to perceive the challenges in each task. There are also quick feedbacks, points, badges, and leader boards suitable to different genres of players to show a level of competence along with a level of task status. Introduction section together with methods of application and playing is available for players to read and understand prior to implementation.

\section{$6 \quad$ Materials and Methods}

The study followed an experimental methodology as best suited to the objectives of this paper. The aim also included the development of a DMG system as well as the enhancement on students' learning performance and motivation toward cultural handweaving art and history in Thailand. To evaluate the effectiveness of the proposed approach, an experiment was conducted with undergraduate students in the Prince of Songkla University.

\subsection{Instruments}

The research instrument was comprised of pre and post-test of the game, a multiplechoice questionnaire was used to evaluate students' knowledge. It contained 10 questions with four possible answers to each. The 10 questions were on southern weaving art, culture and history, and each player had to answer both prior and after the game playing. An assessment test was constructed and consisted of closed-ended statements. The participants were asked to choose an answer from a 5-point Likert scale.

The design of assessment tool was based on the research framework (Figure 1) in regard to aspects of usability, usefulness, and engagement. Each questionnaire item was on 5-point Likert scale (from $1=$ strongly disagree to $5=$ strongly agree). First, the students were asked to play the game (they had 10 minutes to play without limitation of frequencies, so they could repeat it over and over). Later, the students filled out the questionnaire and, eventually, the data were analysed.

\subsection{Participants}

A probability (purposive) sampling approach was used. Participants were chosen among undergraduate students of the Prince of Songkla University. The study sample was 28 students, age 19-22 years old; 12 male and 16 female. 


\subsection{Experimental procedure}

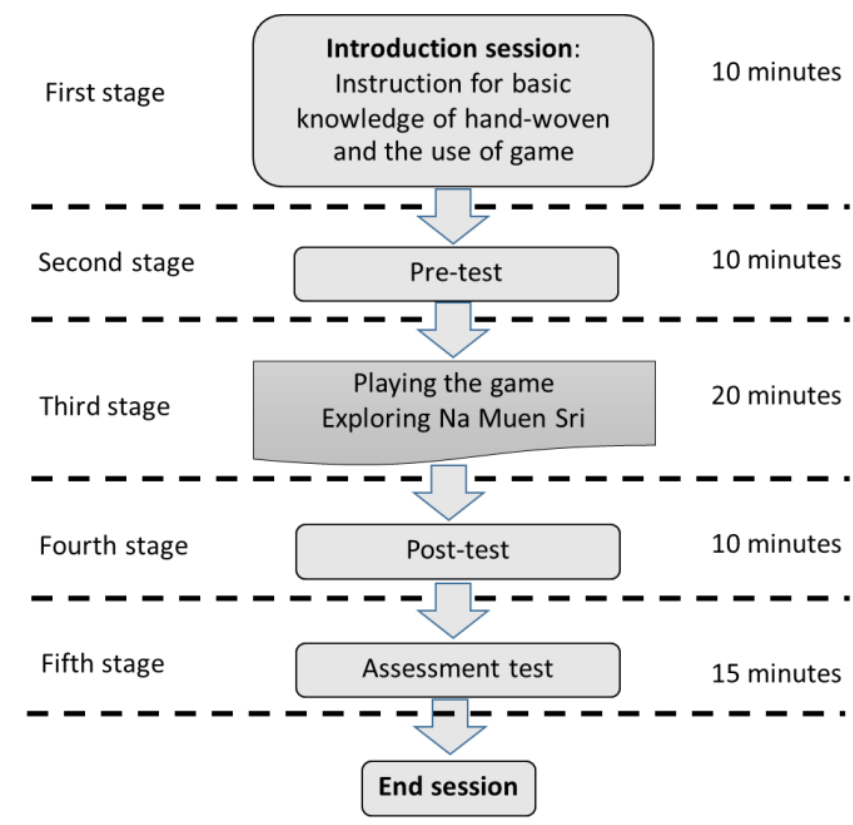

Fig. 3. Experiment procedure

The experiment process was conducted during October and November 2019, and the participants were divided into smaller groups, according to the capacity of the computer lab where the study was implemented. In Figure 3, the procedure of the experiment consists of five stages. (1) In the first stage, an introductory session was held to provide a concise introduction about the game's concept, objectives, how to access it, usage instructions, and what the participants were expected to explore. Students learned the basic knowledge of hand-weaving art and history and received instructions for game playing. (2) Following that, the students took the pre-test for 10 minutes to analyse their knowledge of Na Meun Sri History and art before participating in the learning activity. (3) In the third stage, the students were arranged to play and learn with the game which lasted for 20 minutes. (4) After the learning activity, students took a post-test. (5) Finally, they were asked to fill in the post-questionnaire for game assessment test. Statistical analysis was conducted. A descriptive statistical analysis through frequency distribution, percentages, means, and standard deviations of responses was performed for each questionnaire statement. The descriptive analysis was aimed to provide explanations of the participants' demographic information, as well as to gain general insights into how the DMG influenced their attitudes toward the cultural learning of national hand-weaving art and history. 


\section{$7 \quad$ Results and Discussion}

\subsection{Learning assessment}

The assessment results shown in Table 1 demonstrate that, at the post test, the sampling group possessed improved knowledge of hand weaving art and history: the mean of pre-test was 5.07; the one of post-test was 7.71. The comparison on the learning coefficient reveals that the result of the post test is higher than the pretest at the significance level 0.05. A Cronbach's Alpha reliability analysis was conducted based on the collected questionnaires, and the Cronbach's Alpha was .904, indicating that this questionnaire had internal consistency to a certain extent.

Table 1. The assessment result

\begin{tabular}{|l|c|c|c|c|c|c|}
\hline & N & Mean & S.D. & t & df & Sig \\
\hline Pre-test & 28 & 5.07 & 1.40 & 11.72 & 27 & $0.00^{* *}$ \\
\hline Post-test & 28 & 7.71 & 1.32 & & & \\
\hline
\end{tabular}

\subsection{Evaluation on satisfaction}

Since this study was a preliminary evaluation, a preliminary descriptive statistical analysis was simply conducted based on the data.

The evaluation on players' satisfaction, in regard to usability aspects [31], [32], for optimized game genre was on content design and ease-of-use, such as the level of game playing from easy to more difficult, contents being properly divided and suitably added into the mechanism, the availabilities of feedback system, rules and rewards, etc. The design on aesthetics was emphasized for positive experience and suitability for playing the game e.g. colour, sound, terminology and language, etc., as well as for proper technology and the game development software.

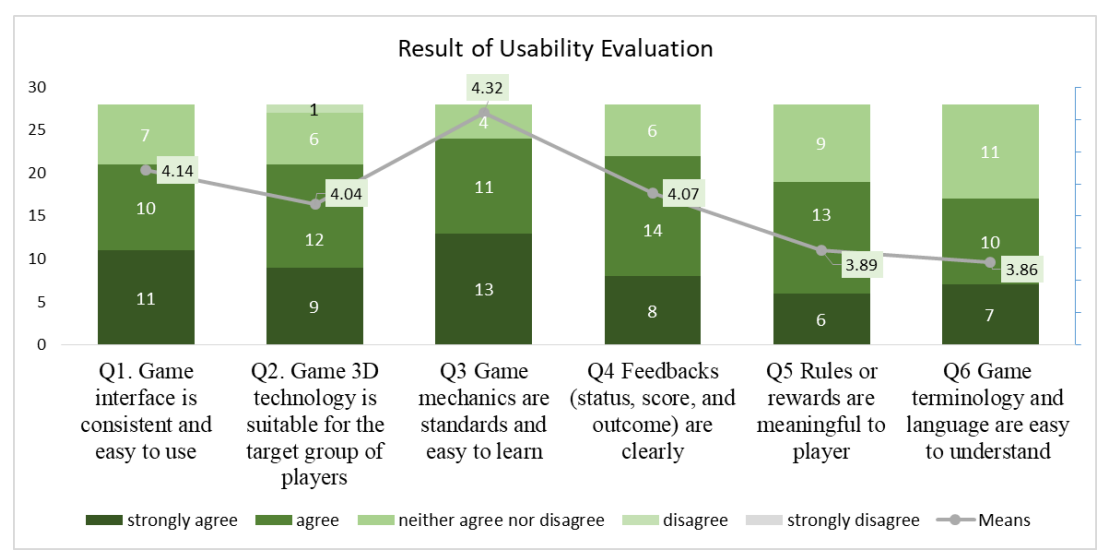

Fig. 4. The usability evaluation 
Most students in the sampling group agreed that the game was easy-to-use (Figure 4) as it shows on the assessment mean of the first three satisfaction aspects: (1) game mechanics are easy to learn (Q3); (2) user interfaces are easy to understand (Q1); and, (3) the game feedback and other information (such as status, score) are clearly presented (Q4). The results disclose that most students can understand the game mechanism and its interface. Also, the game system provides clearly and easily understanding on the presentation of contents. Nevertheless, the results additionally reveal that some students could not comprehend the definitions or terminology and language used in this game story, indicating that there is still room for improvement by re-designing the terminology for easy-to-understand language that is written from the player's point of view.

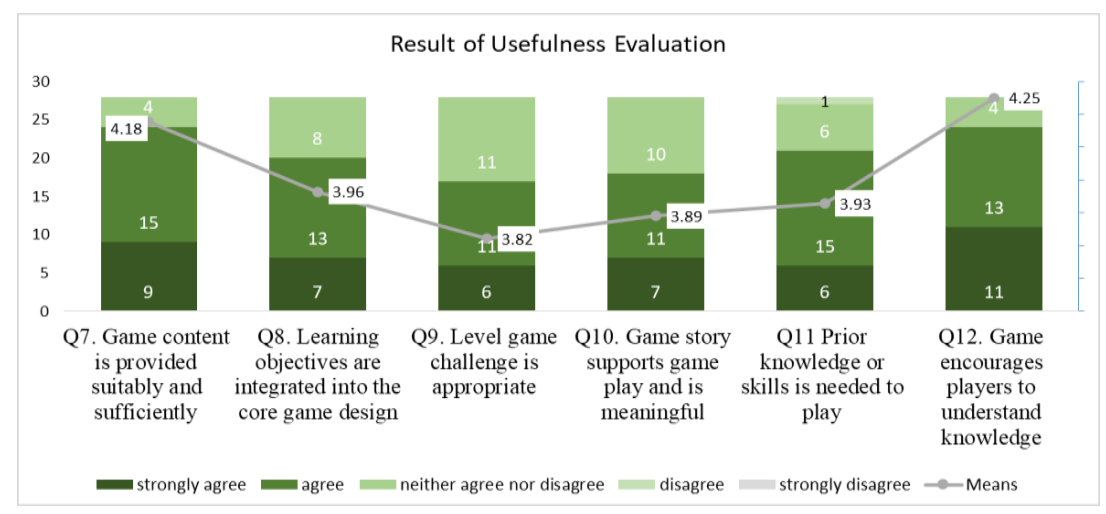

Fig. 5. The usefulness evaluation

The assessment on players' satisfaction for an aspect of perceived usefulness focused on the design of optimized content and game story to be in line with its objective or target [31], [32]. In designing the game challenge to be consistent with the targeted group, prior knowledge or skill was expected to drive or motivate players to understand the knowledge being communicated. The assessment results show the mean of the first top three statements, agreed by the students, that they are on average high, as shown in Figure 5, and such statements are (1) the game could help them understand knowledge of hand-weaving art and history (Q12); (2) the content in the game story is suitable and sufficient (Q7); and (3) learning objectives are integrated into the core game design (Q8). These results demonstrate that players understand the story in the game and a design of the game being aligned with the playing and the learning content can lead to a more effective game. However, they think this game is less challenging (too difficult or too easy) indicating that too-easy or too-difficult game can quickly lose the player's interest, either through boredom or frustration. Therefore, in designing the games, the required level of learning objectives must be integrated with a suitable level of challenge.

As for the satisfaction on engagement, the assessment was focused on factors designed for the players to quickly understand and become part of the game [31], [32]. Designing the game to challenge players' skills requires to have them understand the task and how to succeed it. The game is constructed for players to have enticing 
experiences and to be impressed the first time they play it. Also, the game is a teaching medium, one in which the content is transformed to make it more interesting and simultaneously creating an entertaining atmosphere that further leads to a fun time. Most of all, the game is designed to be used as a self-learning medium.

The evaluation results, as agreed by the students (Figure 6), show the players' satisfaction in a form of mean and the top first three statements are (1) the game is more interesting than the traditional materials (Q17); (2) the game is the new self-learning medium (Q20); and, (3) players have a good interaction with the game (Q18) or the game creates a more fun learning atmosphere. The results indicate that students have positive attitudes to a certain degree toward the game engagement and motivation. The students are comfortable to learn about cultural hand-weaving art and history through the digital MG and how it fits for self-learning although they feel uncomfortable to begin this game for the first time as they thought this game lacks a balance between the player's skills and the game challenges. Therefore, this game is thought to be re-designed by taking into account the interaction between player features and game mechanics. The aforementioned can be used to improve in designing this kind of game in the future.

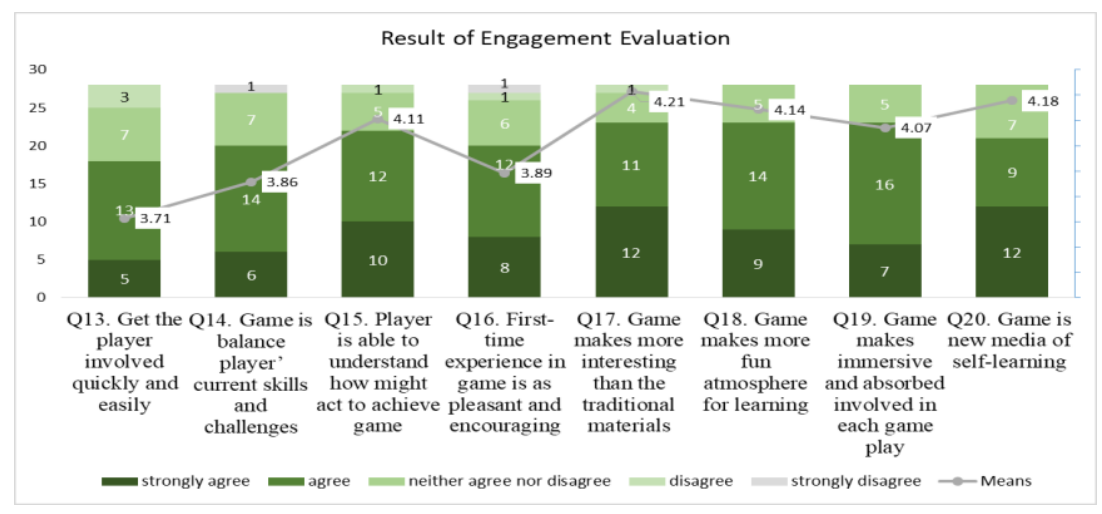

Fig. 6. The engagement evaluation

\section{Discussion and Conclusion}

This study involved the game development for hand weaving fabric art and culture in Na Muen Sri, an area in southern peninsular Thailand. The designed game is called "Exploring Na Muen Sri". The research attention is on the survival of culture in terms of traditional craftsmanship and that the learning could play a role to guarantee a future to the endangered cultural and traditional craftsmanship.

Technology is also employed for better education of arts and cultures explain that utilizing technological simulation for education of arts and cultures can change the content presentation format as there is no need to be at the real exhibition or the actual venue, not to mention that it reduces the risk of venue wreckage. Since using the technological simulation does not create an activity of learning participation among players, DG is brought in, similar to various prior studies [7], [33], [34]. The DG for learning 
support in terms of participation and simulation is considered a technology constructing interaction among learners, learners' behaviours, and knowledge, especially when such learning can lead to motivation and challenges. The young people tend to spend more time on game playing than on learning in traditional formats, and apart from having fun with the activity, it is possible to embed knowledge in a game, consistent with prior studies [6], [8], [35]-[39].

DMC perceptions together with Person-Artefact-Task Model are integrated in this study in designing and developing "Exploring Na Muen Sri". This is because the DMG provides a more time-efficient and budget-friendly alternative than the more complex serious games .Furthermore, thanks to their simplicity and ease of use, the DMGs can be played by hard-core gamers as well as by casual gamers of all ages and are an efficient tool for reaching a broad public. Although a DMG can become popular, it is found that it needs systems and guidance for how content should be delivered across the DMG, and how to create and build the immersive DMG for young people to explore and maintain cultural art and culture. It needs resources to obtain greater exposure to information, gain a comprehension of the local and cultural hand-weaving art and history as well as to promote knowledge of, and interest in cultural and traditional handicraft, in children and young people.

The results of game development and assessment disclose that the game effectively creates perceptual production and awareness in the art and culture, and players gain knowledge through linking places, environment, local culture, art and handicraft. The results on DMG efficiency, similarly, in terms of usability, usefulness and engagement reveal that, overall, the players expressed a high level of satisfaction, whereas regarding the game situation aspect, the players were satisfied with the storytelling and conversations that offer historical content in the format of character design, as all players agreed that such design has an effect in game playing. As the game development is a storytelling of history, there is a limitation in creating comprehension on the characters' roles and features as the players find that the orientation of the characters' roles and tasks is insufficient, making the players' entry into the story slow. In contrast, the players feel that a design of the background is good and suitable with the feature of Na Muen Sri Community area at that time. Likewise, they also express their high satisfaction toward the system design as the game efficiency enables to not only attract players but also stimulate the learning motivation on art, culture and history, which is because the game is fun and contains the drive to motivate the learning, not to mention that players can participate through the nature of the game. The game additionally responds to the basic need in creating the learning environment while setting interesting learning format. A digital game can increase effective learning as its content development is from a simulation of an area environment at that time. Telling story through simulation is a basic part of designing the game, which is a creation of new learning culture that is in line with the young people's behaviours and interests. Thus, "Exploring Na Muen Sri" is a format of creating cultural learning and knowledge transmission through digital technology of a digital game that transforms the format of traditional storytelling into a multimedia and opens opportunities to players to touch the real world in the form of a simulated one. 
There are several topics that can be explored in future studies. In this paper, DMG approach was developed for cultural learning of hand-weaving art and history, while future research can design substantial learning content for other cultural subjects. In addition, the DMG approach could be applied to diverse populations including adult learners. It is further suggested that collecting interview data from students provides better and more in-depth understanding of their perceptions .Researchers could consider integrating DMG approach into mobile devices to create the contextual ubiquitous learning activity and environment.

\section{$9 \quad$ References}

[1] S. Chai-Arayalert and S. Puttinaovarat, "Designing mangrove ecology self-learning application based on a micro-learning approach," Int. J. Emerg. Technol. Learn., vol. 15, no. 11, pp. 29-41, 2020, https://doi.org/10.3991/ijet.v15i11.12585

[2] F. Bellotti, R. Berta, and A. De Gloria, "Designing effective serious games: Opportunities and challenges for research,” Int. J. Emerg. Technol. Learn., vol. 5, no. SPECIAL ISSUE 2, pp. 22-35, 2010, https://doi.org/10.3991/ijet.v5s3.1500

[3] We Are Social, "Digital 2020: Global Digital Overview (Digital Thailand 2020)," Houston USA, 2020.

[4] Youth Radio and Media and Thai Health Promotion Foundation, "Situation of Thai Children Online Gaming Behaviour Survey," Bangkok, 2019.

[5] S. Chai-Arayalert and K. Suttapong, "Increasing Potential of Distribution Channels for Creative Thai Hand-Woven Textile Products in the Digital Economy," Creat. Stud., vol. 13, no. 2, pp. 477-493, Sep. 2020, https://doi.org/10.3846/cs.2020.11865

[6] J. Froschauer, M. Arends, D. Goldfarb, and D. Merkl, "A serious heritage game for art history: Design and evaluation of ThIATRO," in Proceedings of the 2012 18th International Conference on Virtual Systems and Multimedia, VSMM 2012: Virtual Systems in the Information Society, 2012, pp. 283-290, https://doi.org/10.1109/vsmm.2012.6365936

[7] F. M. Dagnino, F. Pozzi, E. Yilmaz, N. Grammalidis, K. Dimitropoulos, and F. Tsalakanidou, "Designing Serious Games for ICH education," in 2015 Digital Heritage International Congress, Digital Heritage 2015, 2015, pp. 615-618, https://doi.org/10.1109/digitalheritage.2015.7419581

[8] C. H. Huang and Y. T. Huang, "An annales school-based serious game creation framework for Taiwanese indigenous cultural heritage," J. Comput. Cult. Herit., vol. 6, no. 2, 2013, https://doi.org/10.1145/2460376.2460380

[9] M. Blades, F. C. Blumberg, and C. Oates, "The importance of digital games for children and young people," Zeitschrift fur Psychol. / J. Psychol., vol. 221, no. 2, pp. 65-66, 2013, https://doi.org/10.1027/2151-2604/a000132

[10] A. G. Salman and C. Antonius, "Interactive educational game, an android mobile app for children learning alphabets," Libr. Hi Tech News, vol. 34, no. 5, pp. 20-22, 2017, https://doi.org/10.1108/lhtn-04-2017-0021

[11] R. Van Eck, "Digital Game-Based Learning: It's Not Just the Digital Natives Who Are Restless," Educ. Rev., vol. 14, no. 2, pp. 16-30, 2006, doi: 10.1145/950566.950596.

[12] S. S. VanDeventer and J. A. White, "Expert behavior in children's video game play," Simul. Gaming, vol. 33, no. 1, pp. 28-48, 2002, https://doi.org/10.1177/1046878102033001002 
[13] M. Hutchison and T. Tin, “'In-Your-Pocket' and 'On-the-Fly' Meeting the Needs of Today's New Generation of Online Learners with Mobile Learning Technology," in The Theory and Practice of Online Learning, T. Anderson, Ed. Canada: AU Press, 2008, pp. 201-220.

[14] K. R. Hamlen, “Children's choices and strategies in video games,” Comput. Human Behav., vol. 27, no. 1, pp. 532-539, 2011, https://doi.org/10.1016/j.chb.2010.10.001

[15] M. Dondlinger, "Educational Video Game Design: A Review of the Literature," J. Appl. Educ. Technol., vol. 4, no. 1, pp. 21-31, 2007.

[16] M. Prensky, "Digital game-based learning," Comput. Entertain., vol. 1, no. 1, p. 21, 2003, doi: 10.1145/950566.950596.

[17] S. M. Alessi and R. T. Stenley, Multimedia for Learning. Methods and Development. Boston, MA: Allyn and Bacon, 2001.

[18] Y. R. Shi and J. L. Shih, “Game Factors and Game-Based Learning Design Model,” Int. J. Comput. Games Technol., vol. 2015, pp. 1-15, 2015, doi: 10.1155/2015/549684.

[19] H. Lukosch, S. Kurapati, D. Groen, and A. Verbraeck, "Microgames for Situated Learning: A Case Study in Interdependent Planning," Simul. Gaming, vol. 47, no. 3, pp. 346-367, 2016, https://doi.org/10.1177/1046878116635468

[20] F. Bellotti, R. Berta, A. De Gloria, and V. Zappi, "Exploring gaming mechanisms to enhance knowledge acquisition in virtual worlds," in Proceedings - 3rd International Conference on Digital Interactive Media in Entertainment and Arts, DIMEA 2008, 2008, pp. 77-84, https://doi.org/10.1145/1413634.1413653

[21] C. Brom, M. Preuss, and D. Klement, "Are educational computer micro-games engaging and effective for knowledge acquisition at high-schools? A quasi-experimental study," Comput. Educ., vol. 57, no. 3, pp. 1971-1988, 2011, https://doi.org/10.1016/j.compedu.2011. $\underline{04.007}$

[22] P. A. Smith and A. Sanchez, "Mini-Games with Major Impacts," in Serious game design and development: Technologies for training and learning, IGI Global Publications, 2010, pp. 1-12. https://doi.org/10.4018/978-1-61520-739-8.ch001

[23] C. Brom, D. Levčík, M. Buchtová, and D. Klement, "Playing educational micro-games at high schools: Individually or collectively?" Comput. Human Behav., vol. 48, pp. 682-694, 2015, https://doi.org/10.1016/j.chb.2015.02.025

[24] M. Z. Nor Azan and S. Y. Wong, "Game based learning model for history courseware: A preliminary analysis," in Proceedings - International Symposium on Information Technology 2008, ITSim, 2008, vol. 1, https://doi.org/10.1109/itsim.2008.4631565

[25] L. Hanes and R. Stone, "A model of heritage content to support the design and analysis of video games for history education,” J. Comput. Educ., vol. 6, no. 4, pp. 587-612, 2019, https://doi.org/10.1007/s40692-018-0120-2

[26] C. M. Finneran and P. Zhang, "A person-artefact-task (PAT) model of flow antecedents in computer-mediated environments,” Int. J. Hum. Comput. Stud., vol. 59, no. 4, pp. 475-496, 2003, https://doi.org/10.1016/s1071-5819(03)00112-5

[27] A. Christopoulos, M. Conrad, and M. Shukla, "Increasing student engagement through virtual interactions: How?" Virtual Real., vol. 22, no. 4, pp. 353-369, 2018, https://doi.org/ 10.1007/s10055-017-0330-3

[28] R. M. Ryan and E. L. Deci, "Self-determination theory and the facilitation of intrinsic motivation, social development, and well-being," Am. Psychol., vol. 55, no. 1, pp. 68-78, 2000, https://doi.org/10.1037/0003-066x.55.1.68

[29] B. Cowley, D. Charles, M. Black, and R. Hickey, "Toward an understanding of flow in video games," Comput. Entertain., vol. 6, no. 2, 2008, doi: 10.1145/1371216.1371223. 
[30] J. I. Rotgans and H. G. Schmidt, "Situational interest and academic achievement in the active-learning classroom,” Learn. Instr., vol. 21, no. 1, pp. 58-67, 2011, https://doi.org/10. 1016/j.learninstruc.2009.11.001

[31] N. Faizan, A. Löffler, R. Heininger, M. Utesch, and H. Krcmar, "Classification of evaluation methods for the effective assessment of simulation games: Results from a literature review," International Journal of Engineering Pedagogy. 2019, https://doi.org/10.3991/ijep.v9i1.9948

[32] Z. Y. Liu, Z. A. Shaikh, and F. Gazizova, "Using the concept of game-based learning in education,” Int. J. Emerg. Technol. Learn., vol. 15, no. 14, pp. 53-64, 2020, https:// doi.org/10.3991/ijet.v15i14.14675

[33] E. Boyle, T. M. Connolly, and T. Hainey, "The role of psychology in understanding the impact of computer games," Entertain. Comput., vol. 2, no. 2, pp. 69-74, 2011, doi: 10.1016/j.entcom.2010.12.002

[34] M. Mortara, C. E. Catalano, F. Bellotti, G. Fiucci, M. Houry-Panchetti, and P. Petridis, "Learning cultural heritage by serious games," Journal of Cultural Heritage, vol. 15, no. 3. pp. 318-325, 2014, https://doi.org/10.1016/j.culher.2013.04.004

[35] K. Dimitropoulos et al., "Capturing the intangible: An introduction to the i-treasures project," in VISAPP 2014 - Proceedings of the 9th International Conference on Computer Vision Theory and Applications, 2014, vol. 2, pp. 773-781, https://doi.org/10.5220/ $\underline{0004871607730781}$

[36] T. Coenen, L. Mostmans, and K. Naessens, "MuseUs: Case study of a pervasive cultural heritage serious game," J. Comput. Cult. Herit., vol. 6, no. 2, 2013, https://doi.org/10.1145/ 2460376.2460379

[37] A. Doulamis, F. Liarokapis, P. Petridis, and G. Miaoulis, "Serious games for cultural applications," Stud. Comput. Intell., vol. 374, pp. 97-115, 2012, https://doi.org/10.1007/978-3$\underline{642-22907-7 \quad 6}$

[38] D. Christopoulos, P. Mavridis, A. Andreadis, and J. N. Karigiannis, "Using virtual environments to tell the story: 'The battle of Thermopylae,"' in Proceedings - 2011 3rd International Conferenceon Games and Virtual Worlds for Serious Applications, VS-Games 2011, 2011, pp. 84-91, https://doi.org/10.1109/vs-games.2011.18

[39] Y. Cao, R. Klamma, and M. Jarke, "The hero's journey - template-based storytelling for ubiquitous multimedia management," J. Multimed., vol. 6, no. 2, pp. 156-169, 2011, https://doi.org/10.4304/jmm.6.2.156-169

\section{Authors}

Supaporn Chai-Arayalert is a lecturer in the Department of Applied Mathematics and Information Systems within the Faculty of Science and Industrial Technology at Prince of Songkla University, Surat Thani Campus. Her current research interests include knowledge management, Green IT, IT project management, e-commerce, and business information systems.

Supattra Puttinaovarat is an assistant professor at Faculty of Science and Industrial Technology, Prince of Songkla University, Surat Thani Campus. Her research interest includes Geographic Information System, Remote Sensing and Information Technology.

Article submitted 2020-11-11. Resubmitted 2020-12-15. Final acceptance 2020-12-16. Final version published as submitted by the authors. 